\title{
Energy Reduction by Workload Adaptation in a Multi-Process Environment *
}

\author{
Changjiu Xian \\ Department of Computer Science \\ Purdue University \\ West Lafayette, IN 47907 \\ cjx@cs.purdue.edu
}

\author{
Yung-Hsiang Lu \\ School of Electrical and Computer Engineering, \\ Purdue University \\ West Lafayette, IN 47907 \\ yunglu@purdue.edu
}

\begin{abstract}
Reducing energy consumption is an important issue in modern computers. Dynamic power management (DPM) has been extensively studied in recent years. One approach for DPM is to adjust workloads, such as clustering or eliminating requests, as a way to trade-off energy consumption and quality of services. Previous studies focus on single processes. However, when multiple concurrently running processes are considered, workload adjustment must be determined based on the interleaving of the processes' requests. When multiple processes share the same hardware component, adjusting one process may not save energy. This paper presents an approach to assign energy responsibility to individual processes based on how they affect power management. The assignment is used to estimate potential energy reduction by adjusting the processes. We use the estimation to guide runtime adaptation of workload behavior. Experiments demonstrate that our approach can save more energy and improve energy efficiency.
\end{abstract}

\section{Introduction}

Energy reduction is an important design issue in computer systems (special issue in IEEE Computer December 2003). Many techniques [1] have been proposed in the last several years. Among these techniques, dynamic power management (DPM) has been widely studied. DPM saves energy by shutting down hardware components when they are idle. Since shutting down and waking up a component consume energy, only long idle periods can justify such overhead and obtain energy savings. Many studies focus on improving the power manager so that it can predict future idleness accurately. While improving the power manager can effectively reduce the energy during long idle pe-

* This work is supported in part by National Science Foundation Career CNS-0347466 and Purdue Research Foundation. Any opinions, findings, and conclusions or recommendations are those of the authors and do not necessarily reflect the views of the sponsors.

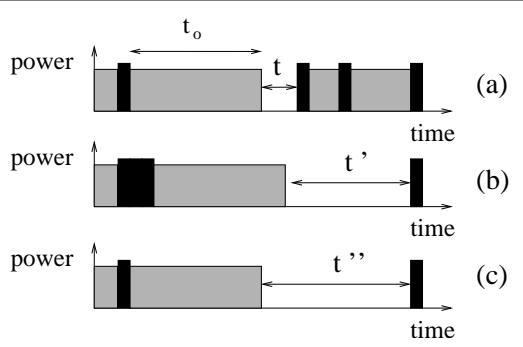

Figure 1. DPM and workload adjustment: (a) shutdown with a timeout, (b) clustering requests, and (c) removing requests.

riods, a workload without long idle periods provides no opportunity for the power manager to save energy. To resolve this, some studies adjust the workload to create long idle periods. These studies can be classified into two major categories: (a) clustering (also called "rescheduling") programs' requests $[2,7,9,10]$ and (b) removing requests $[5,6,11]$. These workload techniques often need to trade off performance and quality of service or use extra resources such as memory buffers. Since unused memory banks can be put into a low-power state [4], using memory for buffering trades off such opportunities. Thus, the workload techniques should only be applied when substantial energy can be saved.

A fundamental question has not been fully addressed: how much energy can possibly be saved by adjusting the workload? We use Figure 1 to illustrate the concept. In the figure, each black bar indicates a request (e.g., a network packet to send or a disk read command). Each gray rectangle means the component (e.g. a network card or a disk) is idle but ready to serve requests. The component consumes energy during idleness. The component can be shut down and consumes no energy. Figure 1(a) shows the original requests and a power manager that shuts down the component after being idle for $t_{o}$. In Figure 1(b), the requests are clustered so the component can remain shut down (also called "sleeping") longer $\left(t^{\prime}>t\right)$ and save more energy. In Fig- 


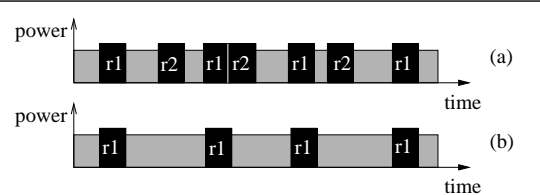

Figure 2. (a) The original requests. (b) Only process 2's requests are removed.

ure 1(c), two requests are removed and the component can sleep even longer $\left(t^{\prime \prime}>t^{\prime}\right)$.

Figure 2 extends the concept to two concurrent processes, $P_{1}$ and $P_{2}$. In this figure, $r_{1}$ and $r_{2}$ represent the requests from $P_{1}$ and $P_{2}$, respectively. If all requests from $P_{2}$ are removed, as shown in Figure 2(b), the idle periods are still short because of $P_{1}$ 's requests. This example suggests that the energy reduction by modifying one process is affected by other processes.

This paper focuses on answering the following two questions: (a) If the requests from a single process are modified by either clustering or removing, how much energy can be saved? (b) When a new process executes or an existing process terminates, how should the other processes respond to obtain more energy savings and better energy efficiency? The efficiency is defined as the ratio of the amount of work to the energy consumption. We assign energy responsibility to individual processes and use the assignments to estimate the potential energy reduction by adjusting each process. The estimation of energy reduction is crucial to run-time adaptation because sufficient energy must be saved to justify the degraded performance. We implement our method in Linux and demonstrate substantial improvements in energy savings and efficiency by providing the estimation to application programs.

\section{Related Work}

Some studies have considered adjusting workloads for power management. Lu et al. [7] order and cluster the tasks for multiple devices to create long idle periods for power management. Weissel et al. [10] propose to assign timeouts to file operations so they can be clustered within the time constraints. Rong et al. [9] divide power management into system and component levels and propose clustering requests by modeling them as stochastic processes. Cai et al. [2] use buffers to cluster accesses to a device. Flinn et al. [5] reduce the quality of service such as the frame size and resolution of video when battery energy is scarce. Zeng et al. [11] assign an energy budget to processes and a process is not allowed to run when its budget is consumed. In a multiprocess environment, the energy savings from adjusting one process can be affected by other processes. Our study provides guidence to the adjustment by estimating the potential energy savings when other concurrently running processes are considered.
We estimate the potential energy savings due to workload adjustment based on the processes' energy responsibilities. PowerScope [6] uses a multimeter to measure the whole computer's power consumption and correlates the measurements to software programs by sampling the program counter. Their study provides information about procedural level energy consumption. Chang et al. [3] conduct a similar measurement with a special hardware called Energy Counter, which reports when a predefined amount of energy is consumed. ECOSystem [11] models the energy consumption of different components individually and assigns energy to the processes by monitoring their usage of individual components. Their study controls processes' energy consumption using operating system (OS) resource allocation. Neugebauer et al. [8] perform similar energy assignment in a system called Nemesis OS providing QoS guarantees. None of these studies examine the relationship between processes' energy responsibilities and the potential energy savings from adjusting the processes. Moreover, they do not examine how energy sharing in a multi-process environment influences the effectiveness of workload adjustment. Hence, these studies are insufficient for estimating the energy savings of workload adaptation in a multiprocess environment.

\section{Estimating Energy Savings}

This section analyzes how energy is consumed in a multi-process environment and estimates the energy saving opportunities from adjusting the workload. We divide energy responsibility between the power manager and the user processes so the processes' energy assignments are independent of specific power management policies. We also divide energy responsibility among the processes based on how they affect the effectiveness of dynamic power management. The assignments are then used to estimate potential energy savings from changing workload. For simplicity, we first assume three power states: busy (serving requests from processes), sleeping (requests have to wait for the component to wake up), and idle (not serving requests but ready to serve without delay). We extend our method to multiple sleeping states in Section 3.3.

\subsection{Energy Responsibility and Sharing}

To determine the opportunity of energy reduction from improving the power manager or adjusting processes, we assign the energy consumption that can be reduced by improving the shutdown accuracy to the power manager and assign the other energy to the user processes. The energy assigned to a process can be reduced by adjusting the process. For example, if the component serves only a single request as shown in Figure 3 (a), the necessary energy consumption includes the wakeup energy $\left(e_{w}\right)$, service energy $\left(e_{a}\right)$, and the shutdown energy $\left(e_{d}\right)$. Any additional energy 


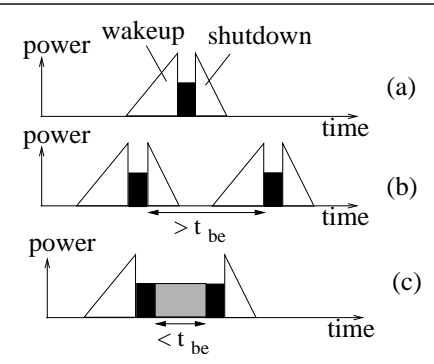

Figure 3. Energy responsibility of a process.

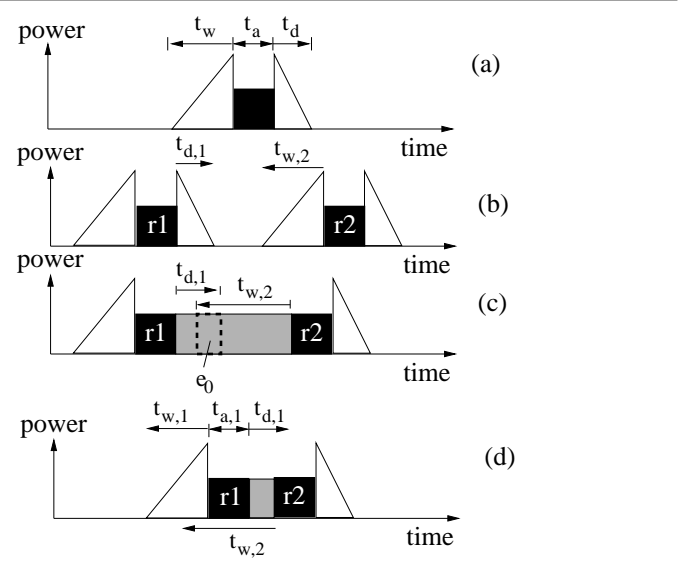

Figure 4. Two requests $r_{1}$ and $r_{2}$ from two processes. (a) Sharing periods. (b) No sharing between $r_{1}$ and $r_{2}$. (c) $r_{1}$ and $r_{2}$ share the energy $e_{o}$ (d) $r_{2}$ shares $e_{w}$ with $r_{1}$ 's $e_{a}$ and $e_{w}$.

can be reduced by performing wakeup and shutdown immediately before and after the service. The energy $e_{w}+e_{a}+e_{d}$ is responsible by the process because it can be reduced only by removing the request. When multiple requests access a component, we can calculate the necessary energy consumption based on the component's break-even time. The break-even time $\left(t_{b e}\right)$ is the minimum duration of an idle period during which the energy saved in the sleeping state can compensate the state-change energy $\left(e_{d}+e_{w}\right)$ [1]. If the idle period is longer than $t_{b e}$ as shown in Figure 3 (b), the processes are responsible for only $e_{d}+e_{w}$ in the idle period. If the idle period is shorter than $t_{b e}$ as shown in Figure 3 (c), the energy in the idle period cannot be reduced by shutdown and such idle energy is assigned to the processes.

We next divide the responsibility among processes to estimate the opportunity for energy reduction from adjusting each process. Suppose the two requests in Figure 3 (b) and (c) are from two different processes. In Figure 3 (b), each process is responsible for $e_{w}+e_{a}+e_{d}$ of its request. In Figure 3 (c), the energy consumption of the two requests are not separated by shutdown because the idle period is less than $t_{b e}$. The processes share energy in this case. To calculate sharing for determining energy responsibility, we first extend the concept from Figure 3 (a). The process is responsible for energy $e_{w}$ before a request and $e_{d}$ after the request. The energy corresponds to time $t_{w}$ and $t_{d}$ as shown in Figure 4 (a); they are called backward sharing period and forward sharing period, respectively. Their values are defined as $\int_{0}^{t_{w}} \rho(t) d t=e_{w}$ and $\int_{0}^{t_{d}} \rho(t) d t=e_{d}$, here $\rho(t)$ is the power at time $t$. The value of $t_{w}$ is calculated backward from the service, i.e., at the moment of the service, $t=0$. In the case of Figure 4 (a), $t_{w}$ and $t_{d}$ are just the component's wakeup delay $\left(\tau_{w}\right)$ and shutdown delay $\left(\tau_{d}\right)$, respectively. In the case of $4(\mathrm{c}), \rho(t)=p_{l}$ (here $p_{l}$ is the idle power) for calculating $t_{d, 1}$ for $r_{1}$ and $t_{w, 2}$ for $r_{2}$, so $t_{d, 1}=e_{d} / p_{l}$ and $t_{w, 2}=e_{w} / p_{l}$.

Two processes do not share energy if their $t_{w}$ and $t_{d}$ do not overlap, as shown in Figure 4 (b). When the idleness between the two requests becomes shorter as shown in Figure $4(\mathrm{c}), t_{d, 1}$ and $t_{w, 2}$ may overlap and the two processes share energy. The energy during the period $t_{d, 1}$ is $e_{d, 1}$ and the energy during the period $t_{w, 2}$ is $e_{w, 2}$. The energy consumption in the overlapped period is $e_{0}$. If $r_{1}$ is removed and no longer responsible for any energy, $e_{o}$ cannot be reduced by any power manager because $r_{2}$ needs energy $e_{w, 2}$ to wake up the component and $e_{w, 2}$ includes $e_{o}$. Similarly, removing $r_{2}$ cannot reduce $e_{o}$ because $r_{1}$ needs energy $e_{d, 1}$ to shut down the component. To reduce the shared energy $e_{o}$, both requests have to be removed. Therefore, both processes are responsible for the overlapped energy $e_{o}$. As the idle period becomes even shorter, $t_{w, 2}$ can overlap with $t_{a, 1}$ and even $t_{w, 1}$, as shown in Figure 4 (d). The rationale of energy sharing is the same - the energy in the overlapped period can be reduced only by removing both processes. We can extend this approach to three or more processes by calculating their sharing periods. If their periods overlap, all processes associated with the overlapping equally share the energy during the overlapped interval.

\subsection{Potential Energy Savings}

Shared energy can be reduced only by adjusting all the sharing processes. Consequently, the potential energy savings from removing only one process' requests are $E_{r}=$ $e_{p}-e_{h}$, here $e_{p}$ is the process' responsible energy and $e_{h}$ is the portion of the process' responsible energy shared with other processes. Clustering a process' requests all together is equivalent to two steps - removing all the process' requests first and then add the cluster back. The cluster consumes energy $e_{w}+\sum e_{a}+e_{d}$, here $\sum e_{a}$ is the sum of the service energy $\left(e_{a}\right)$ of all the clustered requests. Hence, the potential energy savings from clustering a process' requests are $E_{c}=E_{r}-\left(e_{w}+\sum e_{a}+e_{d}\right)$. The energy assigned to the power manager is the potential savings to improve the shutdown accuracy. As a supplement to these potential energy savings, we also calculate the current energy savings $E_{s}$, namely, the energy savings that has been ob- 


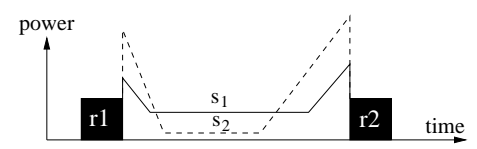

Figure 5. The power consumption of a component with two sleeping states.

tained. This is equal to the difference between the energy consumption without shutdown and the actual energy consumption. For example, for the idle period between the two requests in Figure 3 (b), the consumption without shutdown is $p_{l} \times t_{l}$ (here $p_{l}$ is the idle power and $t_{l}$ is the length of the idle period) and the actual consumption is $e_{d}+e_{w}$. The current energy savings are $E_{s}=p_{l} \times t_{l}-\left(e_{d}+e_{w}\right)$.

\subsection{Multiple Sleeping States}

As processes are only responsible for necessary (or minimal) energy consumption during idle periods, we extend the calculation of necessary consumption to components with multiple sleeping states. Let $s_{1}, s_{2}, \ldots, s_{n}$ be $n$ sleeping states ordered by decreasing power consumption and increasing state-change delay $\left(\tau_{d}+\tau_{w}\right)$ and energy $\left(e_{d}+e_{w}\right)$. Each sleeping state has a corresponding break-even time from the idle state. When the idle period is longer than several break-even times, we need to determine which sleeping state to choose for minimum energy consumption. This state is not necessarily the lower-power sleeping state. Figure 5 is used to illustrate this concept. State $s_{2}$ consumes less power but has larger wakeup and shutdown energy than $s_{1}$. We can calculate the minimum length of an idle period when entering $s_{2}$ saves more energy. Let $t$ be the length of an idle period; $e_{s_{1}}$ and $e_{s_{2}}$ are the state-change energy, $t_{s_{1}}$ and $t_{s_{2}}$ are state-change delay, and $p_{s_{1}}$ and $p_{s_{2}}$ are the power. Using the two states achieves the same energy savings if $e_{s_{1}}+p_{s_{1}}\left(t-t_{s_{1}}\right)=e_{s_{2}}+p_{s_{2}}\left(t-t_{s_{2}}\right)$, or $t=\frac{e_{s_{2}}-e_{s_{1}}+p_{s_{1}} t_{s_{1}}-p_{s_{2}} t_{s_{2}}}{p_{s_{1}}-p_{s_{2}}}$. This is the minimum duration of an idle period to use $s_{2}$. Notice that this is different from $s_{2}$ 's break-even time because $t_{b e}$ is defined between a sleeping state and the idle state, not between two sleeping states. To calculate the sharing periods $t_{w}$ and $t_{d}$ for each request, we use $e_{w}$ and $e_{d}$ of the deepest sleeping state. This is because if there is only a single request, the component should be kept in the deepest sleeping state before and after serving the request in order to consume the minimum energy.

\subsection{Runtime Adaptation}

Previous sections show that, after assigning each process its energy responsibility, we can estimate the potential energy savings from adjusting the workload. In this section, we show that the estimation can be peformed at runtime such that processes can perform runtime adaptation to save energy, improve energy efficiency, or both. Specifically, we periodically calculate and assign energy respon-

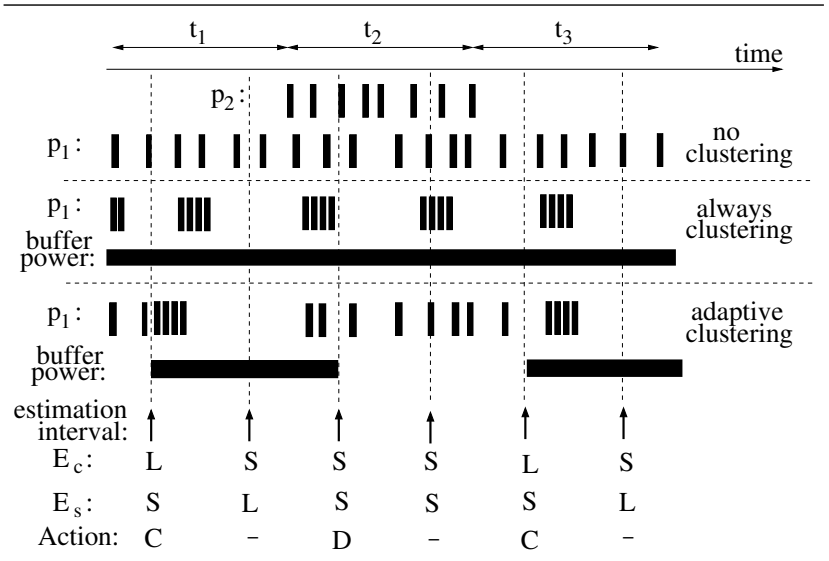

Figure 6. Comparison of clustering schemes when two processes $\left(P_{1}\right.$ and $\left.P_{2}\right)$ running concurrently using the same hardware component. $E_{c}$ : potential energy saving by clustering. $E_{s}$ : current energy saving. $L / S$ : large/small value. $C / D$ : allocating/deallocating the buffer for clustering.

sibility to each process and inform the process of the potential energy savings $E_{c}$ or $E_{r}$ as defined in Section 3.2. We assume that the $E_{c}$ and $E_{r}$ calculated from the previous period can be used to estimate the following period, so we don't need the information of processes' future requests in advance. Processes can combine the estimation and their specific constraints (e.g., performance requirement) to determine when to perform clustering or removing.

We use two techniques as examples of run-time adaptation: (a) Allocating a chunk of memory buffer to cluster a process' requests to a component. For example, additional memory is allocated for an audio/video streaming program to prefetch more frames from the server and the network card is used to fetch frames only when the buffered frames have been consumed. (b) Removing a process' requests by removing or suspending the process. For example, when battery energy is scarce, the user may be willing to terminate or suspend a low-priority program as a tradeoff for energy savings.

Figure 6 illustrates clustering guided by our estimation of potential energy savings. Processes $P_{1}$ and $P_{2}$ generate requests for the same hardware component (e.g., a network card). During $t_{1}$ and $t_{3}$, only $P_{1}$ is running. During $t_{2}, P_{2}$ is running concurrently with $P_{1}$. We assume $P_{2}$ does not allow clustering due to real-time constraints, such as a telephony program transmitting real-time voice data. When only $P_{1}$ is running, clustering $P_{1}$ 's requests can create long idle periods. When $P_{2}$ is also running, clustering $P_{1}$ cannot create long idle periods because $P_{2}$ 's requests are scattered.

The first two rows in Figure 6 show that no clustering technique is used. This case consumes no extra buffer power 
but misses the opportunity to save energy in $t_{1}$ and $t_{3}$ by clustering $P_{1}$ 's requests. The third and fourth rows cluster $P_{1}$ 's requests using buffer throughout the whole duration. Even though energy is saved for the component during $t_{1}$ and $t_{3}$, it does not save energy during $t_{2}$ while additional energy is consumed for the buffer memory. Alternatively, $P_{1}$ can perform adaptive clustering with the information of the potential energy savings $\left(E_{c}\right)$ and the current energy savings $\left(E_{s}\right)$, as calculated in Section 3.2. The value of $E_{c}$ suggests whether it is beneficial to further cluster the requests but does not indicate whether the current running clustering saves energy. In contrast, $E_{s}$ tells how much energy savings we have obtained but does not suggests the potential of further clustering. Consequently, if $E_{c}$ is large, $P_{1}$ should cluster no matter whatever $E_{s}$ is. If buffer has been allocated and both $E_{c}$ and $E_{s}$ are small, $P_{1}$ should stop clustering and release the buffer. This is because the small $E_{c}$ suggests no potential for allocating more buffer for further clustering and the small $E_{s}$ suggests that the current clustering is not beneficial. After the buffer is released, the unused memory banks can enter low-power state.

At the beginning of $t_{1}$, the potential savings are large (shown as "L") and the current savings are small (shown as " $\mathrm{S}$ ") as shown in the figure. Specifically, the "large" or "small" are determined by comparing with the per-period energy consumption of the buffer memory allocated for clustering. As a result, $P_{1}$ clusters the requests. After clustering, the potential savings become small and the current savings become large. When $P_{2}$ starts running at the beginning of $t_{2}$, both $E_{c}$ and $E_{s}$ are small. This indicates that clustering is no longer beneficial so $P_{1}$ stops clustering and releases the buffer. When $P_{2}$ terminates at the end of $t_{2}$, the potential savings become large again and $P_{1}$ should cluster requests during $t_{3}$.

The method of using the estimation to guide runtime workload reduction is similar. Suppose a user would suspend a user process only if the suspension saves substantial energy (e.g., $>10 \%$ ). We can perform runtime adaptation using the estimation $E_{r}$ and the current energy saving $E_{s}$. When $E_{r}$ is large, we suspend the process to save energy. If the process is being suspended and $E_{s}$ is small, the process is resumed to perform more service. Compared to simply terminating the process, this approach can save about the same amount of energy and perform more service. Hence, the energy efficiency is improved.

\section{Experiments}

\subsection{Experimental Setup}

Our prototype uses Integrated Development Platform (IDP) by Accelent Systems running Linux 2.4.18. The IDP uses Intel PXA250 as the processor and provides probing points to measure the power consumption of different hardware components. We install an Orinoco wireless network

\begin{tabular}{|l|l|l|l|}
\hline & meaning & microdrive & wireless \\
\hline$p_{a}(\mathrm{~W})$ & active power & 0.60 & 1.3 tx,, $0.8 \mathrm{rx}$ \\
$p_{l}(\mathrm{~W})$ & idle power & 0.59 & 0.75 \\
$p_{s}(\mathrm{~W})$ & sleeping power & $0.24 / 0.066$ & 0.08 \\
$\tau_{d}(\mathrm{~s})$ & wakeup delay & $0.159 / 0.160$ & 0.03 \\
$\tau_{w}(\mathrm{~s})$ & shutdown delay & $0.273 / 0.716$ & 0.06 \\
$e_{w}(\mathrm{~J})$ & wakeup energy & $0.207 / 0.475$ & 0.079 \\
$e_{d}(\mathrm{~J})$ & shutdown energy & $0.124 / 0.135$ & 0.038 \\
$t_{b e}(\mathrm{~s})$ & break-even time & $0.65 / 1.05$ & 0.15 \\
\hline
\end{tabular}

Table 1. Power parameters. The microdrive has two sleeping states, shown as " $s_{1} / s_{2}$ ".

card and an IBM Microdrive. Table 1 shows the measured parameters of the components used in our experiments. We implemented a Linux kernel module to estimate energy savings. The timing information (e.g., the starting times and the ending times) of the processes' accesses to the devices are collected by modifying the device drivers.

The workload consists of six programs: madplay: an audio player, xmms: an audio streaming program, mpegplayer: an MPEG video player, gzip: a compression tool, scp: a secure file transferring utility, httperf: a program retrieving web pages. These programs have different workload on different components. In our experiments, scp is always running as the background process to upload data files from the Microdrive to a remote server through the wireless network card. We choose scp because it has no stringent timing constraints (like an audio player). The other programs are occasionally selected to execute concurrently with scp. We use the degree of concurrency to indicate how many concurrent user processes are running. When the degree of concurrency is one, only $\mathrm{scp}$ is running. When the degree of concurrency is higher, the other six programs are randomly selected to execute. For example, when the degree of concurrency is three, two other programs execute concurrently with scp. We divide the whole duration of an experment into 300-second intervals and randomly determine a degree of concurrency for each interval. For both clustering and removal, we conduct five experiments with increasing average and maximum degree of concurrency. We perform removal or suspension for $\mathrm{scp}$ on the wireless card and clustering for $\mathrm{scp}$ on the Microdrive. For clustering, we allocate a memory buffer of $10 \mathrm{MB}$ to prefetch data from the Microdrive. The memory consumes $5 \times 10^{-5} \mathrm{~W}$ for every page of $4 \mathrm{~KB}$. The power is calculated using the SDRAM datasheet from the Micron website. This is based on the assumption that unused memory can be turned off to save power [4]. A $0.65 \mathrm{~s}$ timeout is used to shutdown Microdrive and a $0.15 \mathrm{~s}$ timeout for the wirelsess card. 

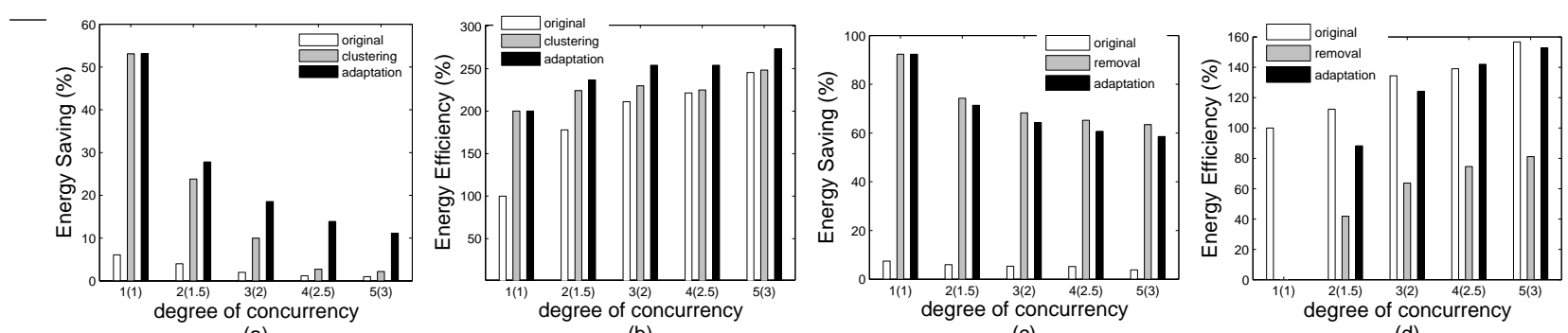

\section{Figure 7. Energy savings and efficiency of clustering and removal for different degrees ${ }^{(\mathrm{d})}$ of concur- rency. Degree $x(y)$ means the maximum(average) degree of concurrency. Higher bars are better.}

\subsection{Energy Savings and Efficiency}

Figure 7 shows our experimental results. Figures 7 (a) and (c) show the energy savings of using clustering and removal for different degrees of concurrency. Figures 7 (b) and (d) show the energy efficiency measured as the number of bytes processed by all programs for every Joule of energy. Figures 7 (b) and (d) are normalized to the original workload as $100 \%$. Figure 7 (a) shows that clustering can save more energy $(47 \%)$ than the original no clustering case when only $\mathrm{scp}$ is running and the degree of concurrency is one. As the degree increases, less energy can be saved by clustering because the concurrent processes create scattered requests. If $\mathrm{scp}$ continues clustering, little energy can be saved by the Microdrive and the network card. Meanwhile, energy is consumed by the buffer memory. In contrast, our adaptive method informs scp to stop clustering and release the buffer memory. Thus, our approach can save more energy and improve energy efficiency. Figures 7 (c) and (d) consider request removal. When the degree of concurrency is one and the requests are removed, over 92\% energy can be saved as shown in Figure 7 (c). However, the efficiency is zero as shown in Figure 7 (d) because no data are copied by scp. As the degree of concurrency increases, $\mathrm{scp}$ can adaptively execute when the microdrive and the network card are in the active state after serving the other programs. Even though the amount of energy saved by adaptation is less than removing scp by up to 5\% as shown in Figure 7 (c), the energy efficiency are significantly higher shown in Figure 7 (d). This is because we resume $\mathrm{scp}$ when the requests from other process are scattered on the network card so the energy during the scattered idleness is utilized to serve scp's requests. The original method (no-removing) has high efficiency but it saves much less energy. The experimental results show the importance of considering concurrent processes to achieve better energy savings and efficiency.

\section{Conclusion}

This paper presents a method to save energy in an environment of multiple processes. We estimate how much energy can be saved when a process clusters or removes requests. By considering the energy sharing among multiple processes, a process can save more energy when it is responsible for most of the energy consumption. If a process is responsible for only a small portion of the energy, the process can stop clustering or removal to improve the energy efficiency. Experimental results indicate that energy savings and efficiency can be significantly affected by the presence of other concurrent processes.

\section{References}

[1] L. Benini and G. D. Micheli. System-Level Power Optimization: Techniques and Tools. ACM TODAES, April 2000.

[2] L. Cai and Y.-H. Lu. Dynamic Power Management Using Data Buffers. In DATE, pages 526-531, 2004.

[3] F. Chang, K. Farkas, and P. Ranganathan. Energy-Driven Statistical Profiling Detecting Software Hotspots. In Workshop on Power-Aware Computer Systems, 2002.

[4] V. Delaluz, M. Kandemir, N. Vijaykrishnan, A. Sivasubramaniam, and M. J. Irwin. Hardware and Software Techniques for Controlling DRAM Power Modes. IEEE Transactions on Computers, 50(11):1154-1173, November 2001.

[5] J. Flinn and M. Satyanarayanan. Energy-Aware Adaptation for Mobile Applications. In ACM SOSP, 1999.

[6] J. Flinn and M. Satyanarayanan. PowerScope: A Tool for Profiling the Energy Usage of Mobile Applications. In IEEE Workshop on Mobile Computing Systems and Applications, pages 2-10, 1999.

[7] Y.-H. Lu, L. Benini, and G. D. Micheli. Low-Power Task Scheduling for Multiple Devices. In International Workshop on Hardware/Software Codesign, pages 39-43, 2000.

[8] R. Neugebauer and D. McAuley. Energy is Just Another Resource: Energy Accounting and Energy Pricing in the Nemesis OS. In Workshop on HotOS, pages 59-64, 2001.

[9] P. Rong and M. Pedram. Hierarchical Power Management with Application to Scheduling. In ISPLED, pages 269-274, 2005.

[10] A. Weissel, B. Beutel, and F. Bellosa. Cooperative IO- A Novel IO Semantics for Energy-Aware Applications. In OSDI, pages 117-129, 2002.

[11] H. Zeng, C. S. Ellis, A. R. Lebeck, and A. Vahdat. ECOSystem: Managing Energy As A First Class Operating System Resource. In ASPLOS, pages 123-132, 2002. 